\title{
Multiscattering illumination in blended acquisition
}

\author{
A. J. Berkhout ${ }^{1}$, G. Blacquière ${ }^{1}$, and D. J. Verschuur ${ }^{2}$
}

\begin{abstract}
In traditional seismic surveys, the firing time between shots is such that the shot records do not interfere in time. However, in the concept of blended acquisition, the records do overlap, allowing denser source sampling and wider azimuths in an economic way. A denser shot sampling and wider azimuths make that each subsurface gridpoint is illuminated from a larger number of angles and will therefore improve the image quality in terms of signal-to-noise ratio and spatial resolution. We show that - even with very simple blending parameters like time delays - the incident wavefield at a specific subsurface gridpoint represents a dispersed time series with a "complex code". For shot-record migration purposes, this time series must have a stable inverse. In a next step, we show that the illumination can
\end{abstract}

be further improved by utilizing the surface-related multiples. This means that these multiples can be exploited to improve the incident wavefield by filling angle gaps in the illumination and/or by extending the range of angles. In this way, the energy contained in the multiples now contributes to the image, rather than decreasing its quality. One remarkable consequence of this property is that the benefits to be obtained from the improved illumination depend on the detector locations in acquisition geometries as well. We show how to quantify the contribution of the blended surface multiples to the illuminating wavefield for a blended source configuration. Results confirm that the combination of blending and multiple scattering increases the illumination energy and, therefore, will improve the quality of shot-record migration results beyond today's capability.

\section{INTRODUCTION}

In traditional seismic acquisition, temporal overlap between shot records is avoided. The consequence is that the source domain is often poorly sampled for economic reasons. In blended acquisition, however, overlap is allowed, resulting in densely sampled, wideazimuth geometries in an economic way (Beasley, 2008; Beasley et al., 2010; Pecholcs et al., 2010). The concept of simultaneous shooting, a special case of blending, is well known from vibroseis acquisition, where lengthy signals are used (sweeps) and optionally coding is applied (Bagaini, 2006). For the marine case, where impulsive sources do not easily allow for signal coding, Beasley et al. (1998) propose to fire such sources simultaneously with large distances between them. See also Vaage (2002), Ikelle (2007), Hampson et al. (2008), and Howe et al. (2008), where temporal jitter between the sources is added optionally. Note that for a densely sampled source configuration, jitter-free simultaneous shooting (equal firing times) becomes plane wave acquisition (Taner, 1976).
Blended acquisition stands for the continuous recording of seismic responses from incoherent source arrays (Berkhout, 2008), the properties of which are characterized by the distances and time shifts between the involved sources, both of which may vary from small to large, e.g., up to several hundreds of meters and several seconds, respectively. Advanced encoding of source signatures is optional. Application of incoherent, multiwavefront source wavefields is a promising development in seismic acquisition and opens new opportunities in imaging ("incoherent migration").

Blended acquisition exchanges aliasing noise for interference noise. This is a very interesting property because, unlike aliasing, interference is not a fundamental problem. Promising results have already been reported on source separation (deblending) by, among others, Akerberg et al. (2008), Spitz et al. (2008), Moore et al. (2008), Huo et al. (2009), Kim et al. (2009), and Lin and Herrmann (2009). In this paper, however, we do not look at source separation, but we concentrate on the illumination properties of a blended

\footnotetext{
Manuscript received by the Editor 24 March 2011; revised manuscript received 26 August 2011; published online 16 February 2012.

${ }^{1}$ Delft University of Technology, Faculty of Civil Engineering and Geosciences, Delft, The Netherlands. E-mail: A.J.Berkhout@ tudelft.nl; gerrit_blacquiere@ hotmail.com.

${ }^{2}$ Delft University of Technology, Faculty of Applied Sciences, Delft, The Netherlands. E-mail: d.j.verschuur@tudelft.nl. (C) 2012 Society of Exploration Geophysicists. All rights reserved.
} 
source configuration. Such knowledge is very important for investigating the potential of incoherent imaging, i.e., imaging without applying deblending.

We distinguish between the illumination and sensing part of an acquisition geometry. Illumination depends on the source geometry and sensing depends on the detector geometry. In this paper, we focus on illumination because we study the effect of blending on the source side. In the case of primary illumination, the illumination part concerns the primary sources only. However, the subsurface is not only illuminated by primary source wavefields (generating the primaries), but also by secondary source wavefields (generating the surface-related multiples). The fact that secondary source wavefields can be used for imaging has been recognized in global seismology. E.g., Bostock et al. (2001) and Rondenay et al. (2005) discussed the improved resolution of regional scale images of the crust and upper mantle of the Earth by including surface-related multiples from teleseismic earthquakes in their imaging method. The use of multiples for velocity estimation and imaging in exploration seismology has been discussed by, among others, Berkhout and Verchuur (1994); Jiang et al. (2005); Schuster (2005); Whitmore et al. (2010); and Verschuur and Berkhout (2011).

In this paper, we consider surface-related multiples as a blended wavefield, the blending being naturally induced. Similar to manmade blending, if we treat the surface-related multiscattering energy correctly, its illumination power can be well exploited. This is important because multiples may illuminate the subsurface from more and other angles than primaries, leading to a higher resolution and a better $\mathrm{S} / \mathrm{N}$. This property applies particularly to wide-azimuth surveys where the near-offset area is generally poorly sampled. We introduce an illumination measure for blended acquisition that includes the illumination properties of the surface-related multiples. Using this measure, the potential benefit of the utilization of multiples can be quantitatively evaluated.

\section{THEORY}

Each temporal frequency component of a seismic data volume can be represented by data matrix $\mathbf{P}\left(z_{d}, z_{s}\right)$, where one column represents a common source gather (shot record), one row represents a common detector gather, and where $z_{d}$ and $z_{s}$ refer to the detector and source depth levels, respectively. See also the Appendix, where extra information on our matrix notation is provided. Matrix element $P_{i j}\left(z_{d}, z_{s}\right)$ is a complex-valued scalar representing one frequency component of the trace related to detector $i$ and source $j$. If we take $z_{d}=z_{s}=z_{0}$, the monochromatic feedback model of

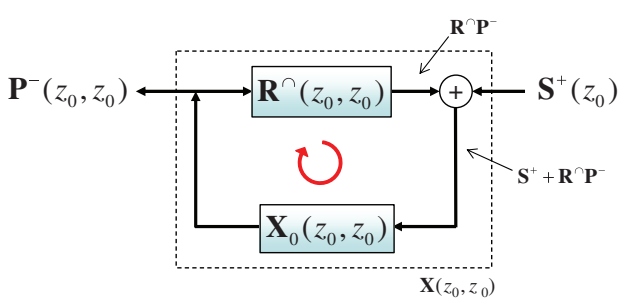

Figure 1. Model for seismic data in the case of a reflective surface (feedback model), showing the double-illumination property. Note that $\mathbf{S}^{+}$is emitting its energy at the source positions (primary illumination) and the energy emitted by the secondary sources, $\mathbf{R}^{\cap} \mathbf{P}^{-}$, is known at the detector positions (secondary illumination). surface seismic data - including internal multiples and surface scattering — is given by (Berkhout, 1982)

$$
\mathbf{P}^{-}\left(z_{0}, z_{0}\right)=\mathbf{X}_{0}\left(z_{0}, z_{0}\right)\left[\mathbf{S}^{+}\left(z_{0}\right)+\mathbf{R}^{\cap}\left(z_{0}, z_{0}\right) \mathbf{P}^{-}\left(z_{0}, z_{0}\right)\right],
$$

where the superscript in $\mathbf{P}^{-}$refers to an upgoing wavefield consisting of primaries and multiples. In source matrix $\mathbf{S}^{+}$, each column represents the downgoing wavefield as generated by a traditional source array like a vibrator group or an airgun array. The individual sources within such an array are located closely together and they are activated (almost) simultaneously. This, in contrast with the sources of a blended source array, which may be far apart and activated with considerable temporal intervals between them. Of course, a column of $\mathbf{S}^{+}$may also represent a single source as in dynamite acquisition. The seismic response of one column of $\mathbf{S}^{+}$ is given by the corresponding column of $\mathbf{P}^{-}$. Matrix $\mathbf{X}_{0}$ is the earth's transfer function without surface-related multiples and matrix $\mathbf{R}^{\bigcap}$ represents the surface reflection for upgoing waves, see Figure 1. In the marine case, i.e., for a flat and stress-free surface, $\mathbf{R}^{\cap}=-\mathbf{I}$, with $\mathbf{I}$ being the unity matrix. Note that $\mathbf{P}^{-}$represents the recorded data without the ghost effect $\left(\mathbf{P}^{+}\right)$. Recently, it was shown that dual streamer technology is very suitable to separate $\mathbf{P}^{-}$and $\mathbf{P}^{+}$(Tabti et al., 2009; Whitmore et al., 2010).

Equation 1 shows that the subsurface is illuminated by primary source wavefields (generating the primaries), as well as by secondary source wavefields (generating the surface-related multiples). We call this phenomenon double illumination. It is important to realize that surface multiples include internal multiple scattering and, therefore, may contain significant illumination power if one or more strong reflectors are present in the subsurface.

An alternative formulation for equation 1 would be $\mathbf{P}^{-}=\mathbf{X S}^{+}$, where $\mathbf{X}$ represents the earth's transfer function with surface-related multiples (Figure 1). Because $\mathbf{X}$ hides the double-illumination property, we strongly prefer the formulation of equation 1 . In addition, $\mathbf{X}$ is much more complex than $\mathbf{X}_{0}$.

In the case of blending, equation 1 becomes

$$
\begin{aligned}
\mathbf{P}^{-}\left(z_{0}, z_{0}\right) \vec{\Gamma}_{j}\left(z_{0}\right)= & \mathbf{X}_{0}\left(z_{0}, z_{0}\right)\left[\mathbf{S}^{+}\left(z_{0}\right)\right. \\
& \left.+\mathbf{R}^{\cap}\left(z_{0}, z_{0}\right) \mathbf{P}^{-}\left(z_{0}, z_{0}\right)\right] \vec{\Gamma}_{j}\left(z_{0}\right),
\end{aligned}
$$

or,

$$
\vec{P}_{j}^{-}\left(z_{0}, z_{0}\right)=\mathbf{X}_{0}\left(z_{0}, z_{0}\right) \vec{Q}_{j}^{+}\left(z_{0}\right) .
$$

Here, vector $\vec{\Gamma}_{j}$ contains the information of blended source array $j$ (Berkhout, 2008) and $\vec{Q}_{j}^{+}\left(z_{0}\right)$ represents the total blended source wavefield that leaves the surface $\left(z_{0}\right)$. The time shifts (or more complex codes) are defined by the complex-valued vector elements of $\vec{\Gamma}_{j}$. Note that in the extreme case of blended acquisition, a seismic survey may consist of one mega-size blended shot-record, indicating that (1) a blended source array may have any size, (2) differential delays may become very large, and (3) recording duration may equal the total survey time.

We now investigate the illumination of a single subsurface gridpoint. We start with the conventional situation, i.e., without blending and without surface scattering. Next, blending is added and finally surface scattering is included as well. Without blending and multiples the incident wavefield in subsurface gridpoint $k$ at 
$\left(x_{k}, y_{k}, z_{m}\right)$, generated by a single traditional source array $j$, can be represented by the complex-valued scalar quantity:

$$
P_{k j}^{+}\left(z_{m}, z_{0}\right)=\vec{W}_{k}^{\dagger}\left(z_{m}, z_{0}\right) \vec{S}_{j}^{+}\left(z_{0}\right) .
$$

The dagger symbol $\dagger$ denotes a row vector and $\vec{W}_{k}^{\dagger}$ equals the $k$ th row of downward propagation matrix $\mathbf{W}^{+}$. Vector $\vec{W}_{k}^{\dagger}$ describes downward extrapolation from all locations at surface level $z_{0}$ to gridpoint $k$ at depth level $z_{m}$. Vector $\vec{S}_{j}^{+}$corresponds to column $j$ of source matrix $\mathbf{S}^{+}$. In seismic shot-record migration, the source-related step aims at transforming $P_{k j}^{+}=\vec{W}_{k}^{\dagger} \vec{S}_{j}^{+}$into unity for all gridpoints at all depth levels $z_{m}(m=1,2, \ldots)$. In other words, this step aims at a deconvolution process that transforms the incident source wavefield at gridpoint $k\left(P_{k j}^{+}\right)$into a bandlimited spike. The illumination strength for the involved frequencies must be sufficient to make this step successful. A proper seismic acquisition design should guaranty this requirement. Moreover, for resolution reasons, it should provide a broad range of temporal and spatial frequencies. Note that in today's practice, this deconvolution process is generally approximated by a cross-correlation process.

Now, the conventional illumination of the subsurface will be improved by adding more sources via source blending, i.e., we do not increase the number of records, but we increase the number of sources per record

$$
P_{k j}^{+}\left(z_{m}, z_{0}\right)=\vec{W}_{k}^{\dagger}\left(z_{m}, z_{0}\right) \mathbf{S}^{+}\left(z_{0}\right) \vec{\Gamma}_{j}\left(z_{0}\right)
$$

Figure 3 shows one snapshot of a blended source array, illustrating that every gridpoint in the subsurface is illuminated from many directions. The illumination can even be further improved by including the illumination properties of the surface multiples as well (Figure 2). We call this double illumination (see also equation 2)

$$
\begin{aligned}
P_{k j}^{+}\left(z_{m}, z_{0}\right)= & \vec{W}_{k}^{\dagger}\left(z_{m}, z_{0}\right)\left[\mathbf{S}^{+}\left(z_{0}\right)\right. \\
& \left.+\mathbf{R}^{\cap}\left(z_{0}, z_{0}\right) \mathbf{P}^{-}\left(z_{0}, z_{0}\right)\right] \vec{\Gamma}_{j}\left(z_{0}\right) \\
= & \vec{W}_{k}^{\dagger}\left(z_{m}, z_{0}\right) \vec{Q}_{j}^{+}\left(z_{0}\right) .
\end{aligned}
$$

Equation 6 is the connection between a blended source array $(j)$ at the reflective surface $\left(z_{0}\right)$ and the corresponding double illumination at a specific subsurface gridpoint $(k)$ in the subsurface $\left(z_{m}\right)$, taking the surface multiples into account.
Now, the source-related step in migration aims at transforming $P_{k j}^{+}=\vec{W}_{k}^{\dagger} \vec{Q}_{j}^{+}$into a unit incident wavefield at each gridpoint $k$ in the subsurface. In other words, it aims at deconvolving for the time series given by $\vec{W}_{k}^{\dagger} \vec{Q}_{j}^{+}$. The angle-dependent information in $P_{k j}^{+}$can be assessed by applying the deconvolution for neighboring gridpoints as well: cross-deconvolution (see next section). The improved spectral amplitude properties of $\vec{W}_{k}^{\dagger} \vec{Q}_{j}^{+}$(see equation 6) with respect to $\vec{W}_{k}^{\dagger} \vec{S}_{j}^{+}$(see equation 4 ) quantify the added value of blended double illumination.

It is important to realize that in a practical implementation, e.g., applying a migration algorithm, the total illuminating wavefield $\vec{Q}_{j}^{+}$ is known for positions where the primary sources are present (given by the source elements in $\mathbf{S}^{+} \vec{\Gamma}_{j}$ ) and for positions where the secondary sources are measured, i.e., for the detector locations given by the elements in $\mathbf{R}^{\cap} \mathbf{P}^{-} \vec{\Gamma}_{j}$. This means that the benefits to be obtained from the secondary illumination depend on the detector distribution of the acquisition geometry. Note that the concept of double illumination shows that improved detector sampling is favorable for secondary P-source illumination. This property is illustrated in Figure 4. In addition, in multicomponent detection (think of ocean-bottom acquisition) the double illumination concept can be potentially extended to secondary S-sources as well: full wavefield double illumination.

It is important to bear in mind that for one shot record the illuminating primary source wavefield in conventional acquisition is generated by one source (array) at one location. In blended acquisition, however, the illuminating primary source wavefield is generated by many sources (or source arrays) at many different positions. Taking into account that shot-record migration has become the industry standard, this is a very important difference for stability reasons (imaging means deconvolving for $P_{k j}^{+}$). Note also that if we consider two surveys, one unblended and one blended and both with the same number of shot records, then the number of involved

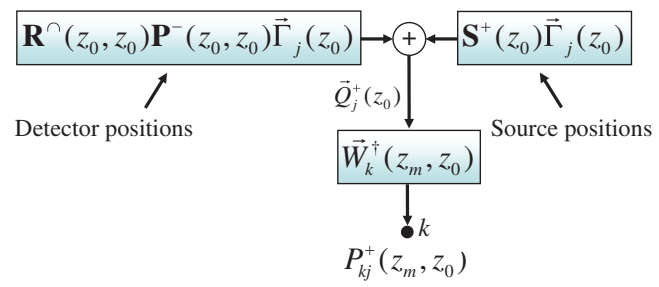

Figure 2. The incident wavefield at subsurface gridpoint $k$ due to blended primary and secondary sources at the surface (blended double illumination).

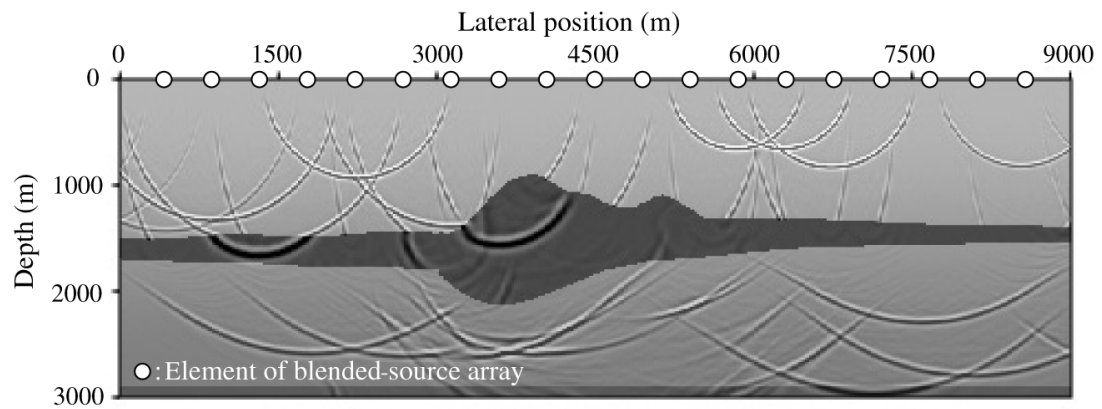

Figure 3. One snapshot of an incoherent wavefield generated by a blended source array at the surface. Every subsurface gridpoint is illuminated by a multiwavefront wavefield from many directions. In blended acquisition, a blended-source array should be judged by its degree of incoherency. 
source positions will be much higher in the blended survey (at least double), leading to improved primary illumination as well as improved stability of the deconvolution process in the imaging step.

\section{CROSS-DECONVOLUTION}

In the data of one blended shot record, each subsurface gridpoint is illuminated from many directions: for a blended-source array with $N$ source elements there are at least $N$ gridpoint-dependent directions. To quantify the spatial bandwidth of this multiwavefront, illuminating wavefield at gridpoint $k$, the cross-deconvolution function is computed (Figure 5)

$$
C_{l k}^{+}(j)=P_{l j} E_{j j}^{-1}(k) P_{j k}^{*},
$$

for one blended shot record, and

$$
C_{l k}^{+}=\sum_{j} P_{l j} E_{j j}^{-1}(k) P_{j k}^{*},
$$

for all involved blended shot records,

where $l$ is ranging around $k$ and $E_{j j}(k)$ equals the frequencydependent energy of the incident wavefield in $k$. It is given by $E_{j j}(k)=P_{k j} P_{j k}^{*}+\epsilon^{2}, \epsilon$ being a small stabilization constant. In equations 7 and $8, P_{l j}$ equals $P_{l j}^{+}$and $P_{j k}^{*}$ is the conjugate-complex of $P_{k j}^{+}$. Figure 5 shows the cross-deconvolution function. It illustrates the value of blended (double) illumination: the more $C_{l k}^{+}(j)$ approaches a band-limited delta function, the better the properties of the incident wavefield (energy, bandwidth) for shot-record migration. Note that in angle-dependent imaging (bifocal imaging), the first term in equations 7 and $8\left(P_{l j}\right)$ is replaced by reflected wavefield $P_{l j}^{-}: P_{l j}=P_{l j}^{-}$. This means that cross-deconvolution of the incident wavefield $\left(P_{l j}=P_{l j}^{+}\right)$becomes normalized crosscorre- lation between incident and reflected wavefield (Berkhout, 1997). Hence, if we assume a reflection coefficient of +1 and we assume no limitations (sampling, aperture) on the detector side, then $P_{l j}^{-}=$ $P_{l j}^{+}$and equations 7 and 8 describe the process of bifocal imaging, showing the potential spatial bandwidth in migrated data. Figure 5 also illustrates that the Fresnel zone is a nonunique concept if we deal with incoherent wavefields: Instead of one wavefront with a unique curvature, an incoherent wavefield contains many wavefronts with many different curvatures.

A further discussion on the migration of blended shot records taking advantage of double illumination is provided by Verschuur and Berkhout (2011).

\section{EXAMPLES}

To illustrate the principle of double illumination, consider the simple three-reflector model shown in Figure 6. The gridpoint under consideration, $k$, is located at $\left(x_{k}, y_{k}, z_{m}\right)=(1000,500,1000) \mathrm{m}$. For a reflection-free surface and a single point source at the origin $(0,0,0) \mathrm{m}$, the incident wavefield at gridpoint $k$ contains the source wavelet that has traveled from the source at $z_{0}=0 \mathrm{~m}$ to gridpoint $k$ at $1000 \mathrm{~m}$, see Figure $7 \mathrm{a}$. The incident wavefield $\left(P_{k j}^{+}=\vec{W}_{k}^{\dagger} \vec{S}_{j}^{+}\right)$has been computed in an area around $k$ (Fresnel zone) and its angular information was determined via cross-deconvolution followed by a linear Radon transform, see Figure 7d. As expected, the incident energy arrives from one angle, which means that angle-dependent reflection information can not be retrieved from this incident wavefield.

Next, a blended array of five sources is considered, oriented in the azimuthal direction. The source locations are shown in Figure 6. Figure $7 \mathrm{~b}$ shows the incident wavefield at gridpoint $k$. Clearly, the arrivals of the five sources can be recognized, the second arrival being a constructive interference of two individual arrivals. Note that much more energy is arriving at gridpoint $k$ than in the no-blending case. Moreover, this energy is arriving from different angles, see Figure 7e. In particular, the range of azimuths is much
Figure 4. The benefits of the secondary illumination can be exploited if detectors are present at the reflecting interface to measure the complex source signature $\left(\mathbf{P}^{+}=\mathbf{R}^{\cap} \mathbf{P}^{-}\right)$. Conventional acquisition is shown on the left, with the number of detectors increasing from (a) to (c). Blended acquisition is shown on the right, with the number of detectors increasing from (d) to (f). For multicomponent detectors, secondary $P$-sources can be potentially supplemented with secondary $S$-sources (think of ocean-bottom acquisition). a)

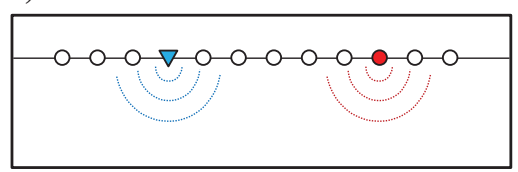

b)

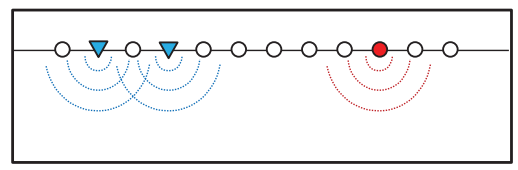

c)

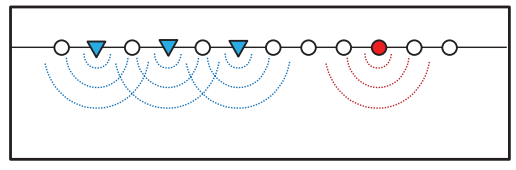

- Primary source, signature simple and known

O Secondary source, signature complex and unknown $\nabla$ Secondary source at detector position, signature complex and known d)

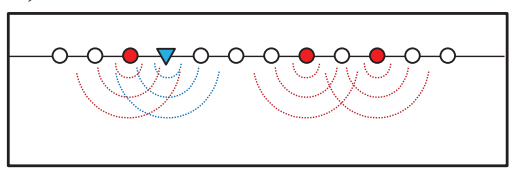

e)

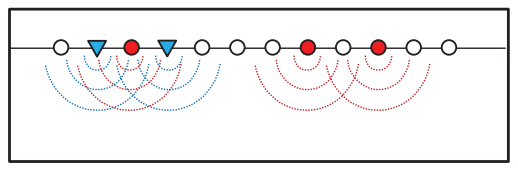

f)

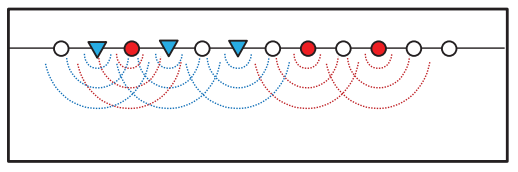


larger. As predicted by the theory, the incident wavefield at gridpoint $k$ has the character of a dispersed time series (containing multioffset, multiazimuth information). The simple, single delay code of the individual sources at the surface has become a complex, multidelay code at the subsurface gridpoint. The spectral properties of this code determine the imaging power of the blended source geometry.

The examples shown represent the situation after perfect surface multiple removal (SRME). We now include surface multiples, representing the situation without SRME. The incident wavefield a)

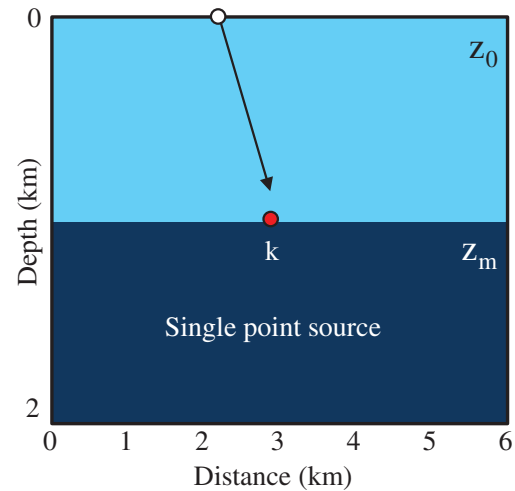

Geometry

d)

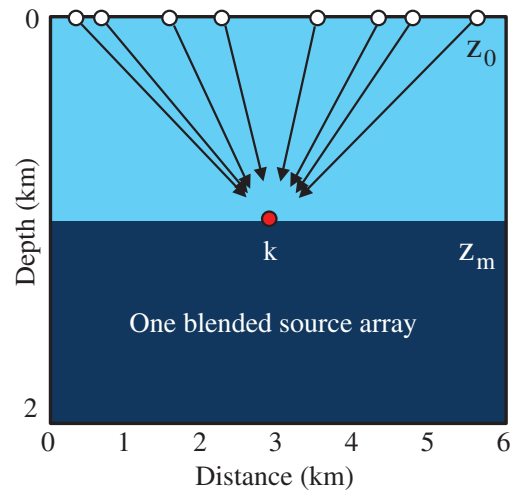

Geometry

g)

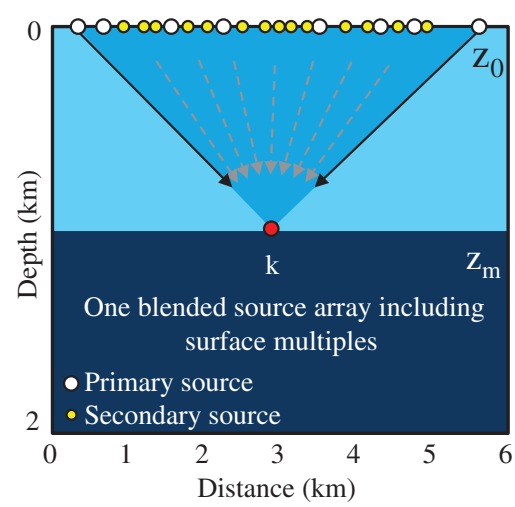

Geometry b)

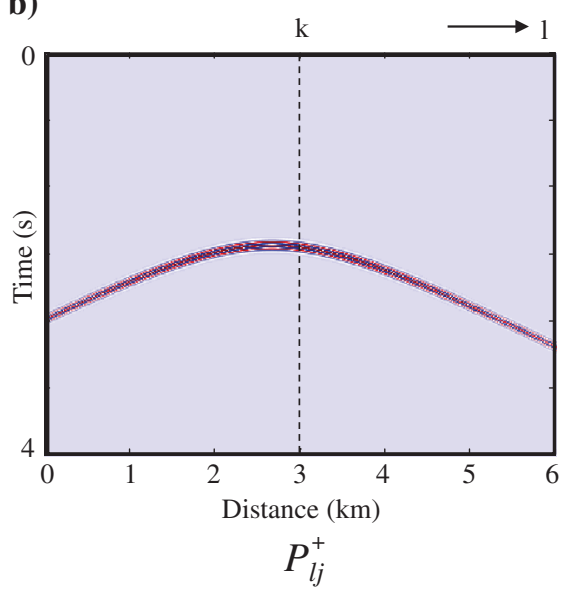

e)

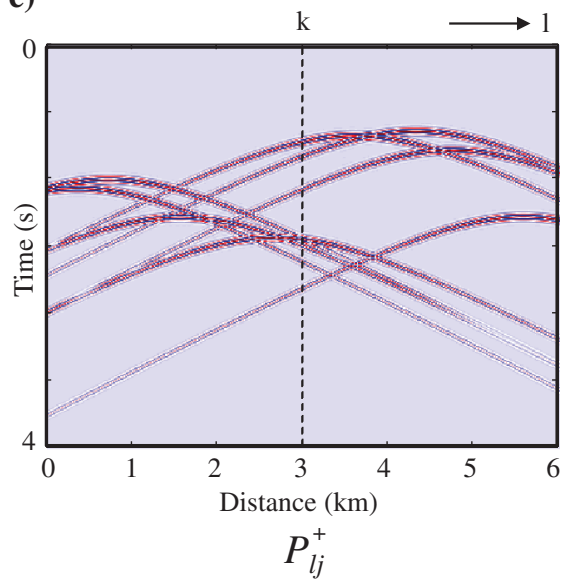

h)

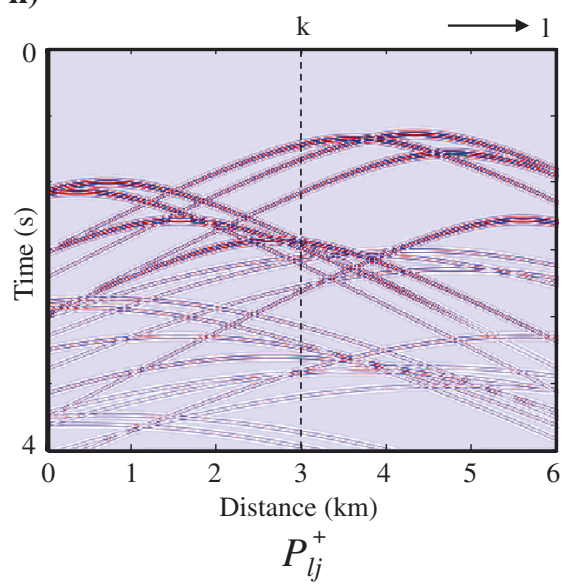

c)

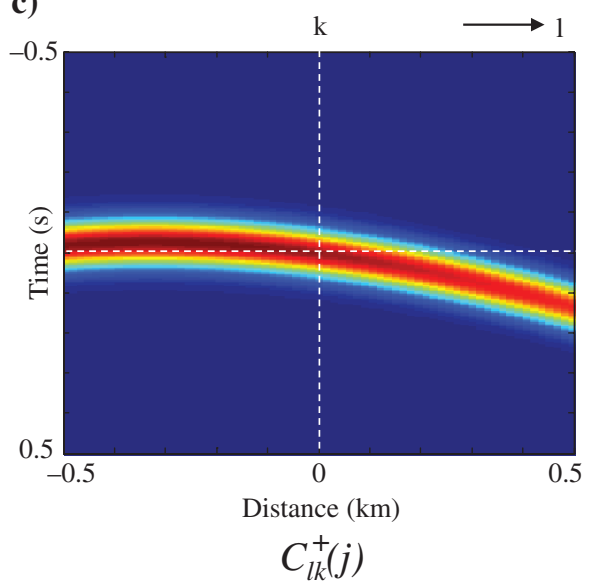

f)

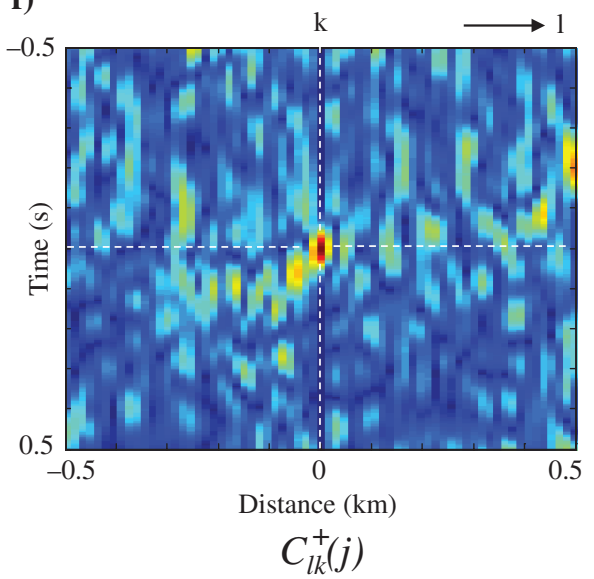

i)

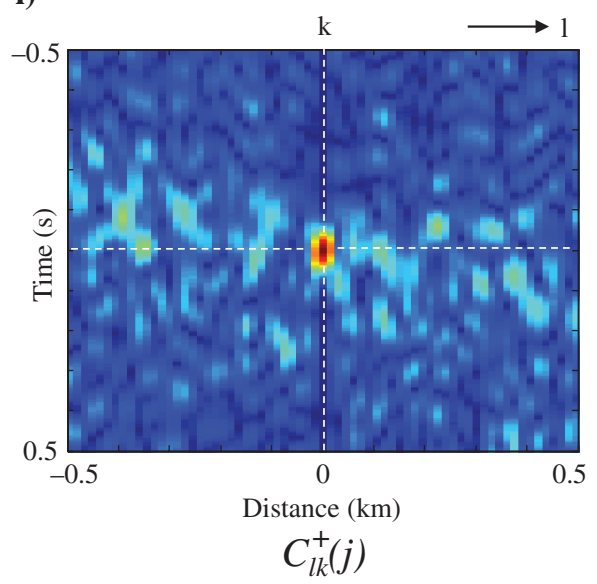

Figure 5. Geometry (a, d, g), incident wavefield at depth $z_{m}(\mathrm{~b}, \mathrm{e}, \mathrm{h})$ and envelope of cross-deconvolution function (c, f, i) around gridpoint $k$ for a single point source $(a, b, c)$, one blended source array (d, e, f) and one blended source array with the surface-multiple-generating secondary sources $(\mathrm{g}, \mathrm{h}, \mathrm{i})$. In practice, $C_{l k}^{+}(j)$ need be summed over all blended-source arrays $(j)$. 


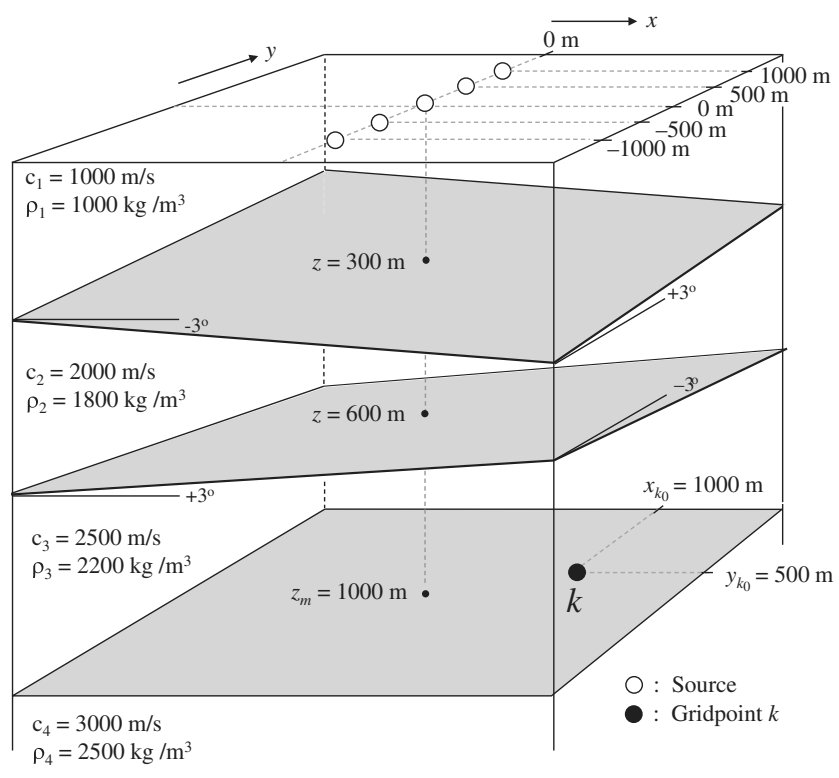

Figure 6. Triple reflector model that is used to illustrate the potential of blended double illumination. Note that the blended source array is oriented in the azimuthal direction.
$\left(P_{k j}^{+}=\vec{W}_{k}^{\dagger} \vec{Q}_{j}^{+}\right)$at gridpoint $k$ is shown in Figure 7c. Several orders of multiples are present, which also illuminate gridpoint $k$. The angles of illumination are shown in Figure 7e. Angles of incidence that were missing in the illumination by the primaries, in particular the smaller angles (related to the missing small offsets), have been provided by the multiples.

In addition to the angular information in Figure 7e, Figure 8a displays the illumination strength, i.e., the energy of the incident wavefield $\left(E_{j j}(k)\right)$ is shown for the three examples. It was obtained by computing the sum of the squared time samples of the incident wavefield. Here, the illumination strength of a conventional seismic experiment (one source) is defined to be $100 \%$, see Figure 8 a (1). When blending is considered, the illumination strength increases to $638 \%$ (2). Note that the illumination strength may vary locally due to space-variant interferences. If surface multiples are included as well, the increase is $721 \%$ (3).

It is important to realize that the contribution of the multiples is nonlinear in the reflection coefficients of the three boundaries. Figure $8 \mathrm{~b}, 8 \mathrm{c}$, and $8 \mathrm{~d}$ show the increase from $721 \%$ to $766 \%$, to $812 \%$, and to $901 \%$, respectively if we multiply the reflection coefficients by a factor of 1.2, 1.4 and 1.8, respectively. Again, the illumination strength is defined to be $100 \%$ for the conventional cases. Of course, the same is achieved if we include more reflectors while keeping the reflection coefficients unchanged.
Figure 7. Left column: the incident wavefield at subsurface gridpoint $k$ for three source configurations; right column: the corresponding crossdeconvolution function $C_{l k}^{+}$in the ray parameter domain (see equation 7). Note the influence of the surface-related multiples (4e to $4 \mathrm{f}$ ). a)

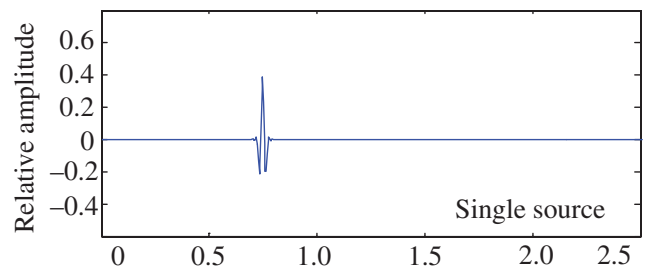

b)

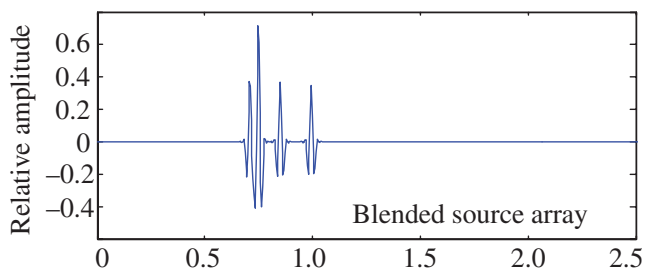

c)

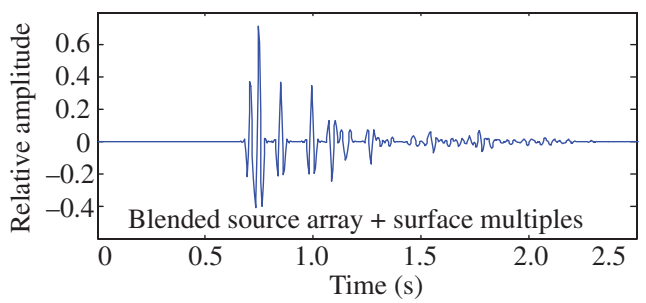

d)

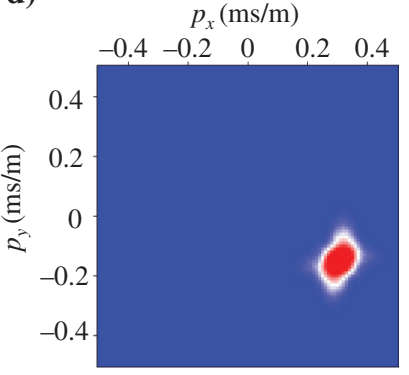

e)

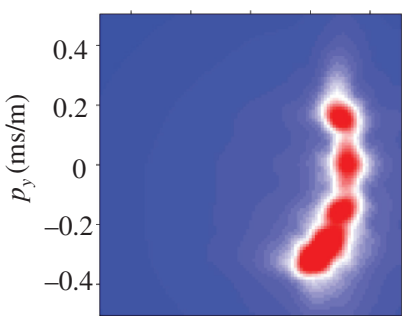

f)

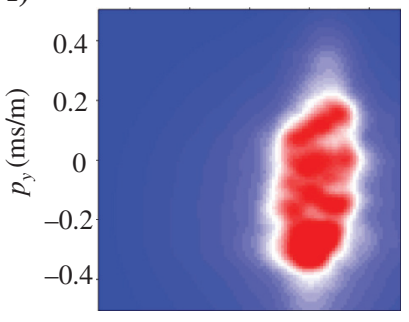


A quantitative measure for the relative contribution of the secondary sources at $z_{0}$ (elements of vector $\mathbf{R}^{\cap} \vec{P}_{j}^{-}$) to the total illuminating wavefield can be indirectly determined by computing for each shot record $(j=1,2, \ldots)$ the energy ratio between the surface multiples a)

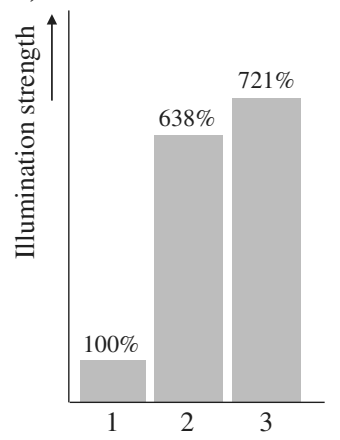

c)

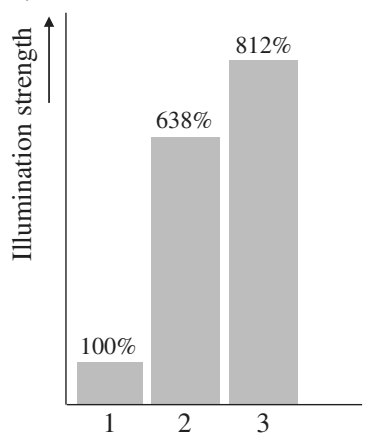

b)

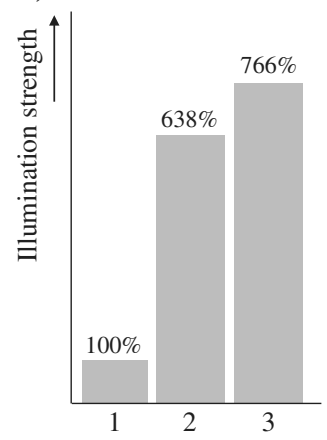

d)

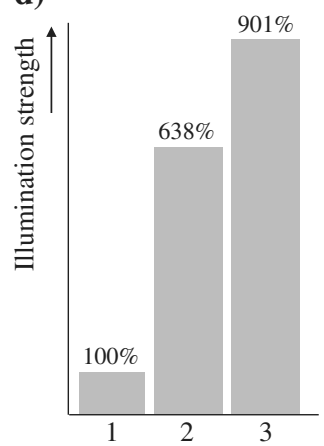

Figure 8. The illumination strength $\left(E_{j j}(k)\right)$ at subsurface gridpoint $k$ for (1) single source, (2) blended source array, (3) blended source array with surface multiples. In Figures $8 b, 8 c$, and $8 d$ the reflectivity has been multiplied by $1.2,1,4$, and 1.8 , respectively with respect to Figure 8a to show the nonlinearity if multiples are included.

a)

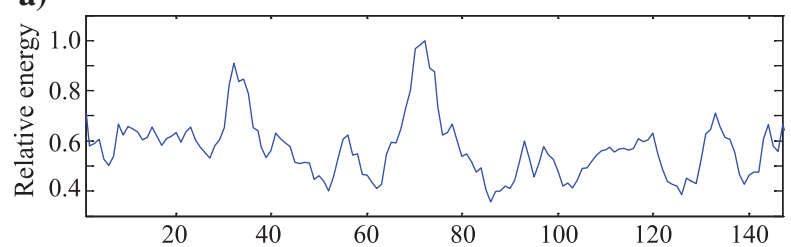

b)

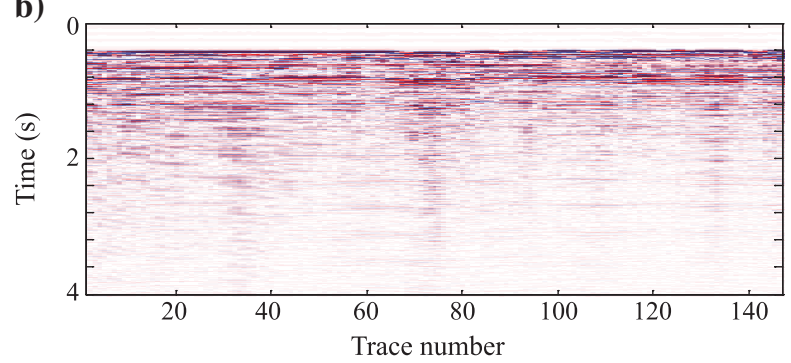

Figure 9. The relative strength of the secondary sources (a) in a North Sea common offset gather (b). In high-reflection areas with strong primaries and many orders of multiples the secondary sources are stronger than in low-reflection areas.
$\left(\mathbf{X}_{0} \mathbf{R}^{\cap} \vec{P}_{j}^{-}\right)$and the primaries $\left(\mathbf{X}_{0} \vec{S}_{j}^{+}\right)$. The larger this ratio, the larger the contribution of the secondary sources $\left(\mathbf{R}^{\cap} \vec{P}_{j}^{-}\right)$to the illumination. Note that if we compute the total energy of individual traces (elements $P_{i j}^{-}$of vector $\vec{P}_{j}^{-}$), i.e., the sum of squares of the time samples, direct information is obtained on the strength of the individual secondary sources. Figure 9 shows this information for a common offset gather of a North Sea line. Clearly, we see that in high-reflectivity areas, the strength of the secondary sources is larger than in areas where reflections (primaries as well as multiples) are weak.

\section{FINAL REMARKS}

The concept of blending shows promise to offer better quality (due to improved illumination) as well as favorable economics (due to efficient survey times). This unique property - more value for money - may explain the huge interest of the industry for this relatively new technology. This paper shows that illumination properties can be further improved by utilizing surface multiple energy (double blended illumination) rather than removing it. Again this can be realized with very attractive economics because surface multiples are free of charge.

In our current research, we investigate the illumination by internal multiples as well. This will provide another "free-of-charge" enhancement of the incident wavefield.

Particularly in areas with an abundance of surface and internal multiple energy, our proposed extended illumination may improve the capability of the seismic method significantly.

\section{CONCLUSIONS}

A blended source wavefield is described by the product of the source matrix and the blending vector: $\mathbf{S}^{+} \vec{\Gamma}_{j}$. Illumination of the subsurface by this multiwavefront wavefield is determined by the blending parameters, i.e., the number and locations of the involved sources, their time delays (or more complex codes), and by the propagation properties of the subsurface: $\mathbf{W}^{+} \mathbf{S}^{+} \vec{\Gamma}_{j}$.

Even with a very simple blending code, e.g., time delays only, we have shown that the incident wavefield at a particular subsurface gridpoint is represented by a dispersed time series, corresponding to a complex code. This time series is gridpoint-dependent and contains multioffset, multiazimuth information.

We also have shown that the primary incident wavefield, $\mathbf{W}^{+} \mathbf{S}^{+} \vec{\Gamma}_{j}$, is enhanced by the secondary sources at the surface that generate the surface-related multiples (blended double illumination): $\mathbf{W}^{+}\left[\mathbf{S}^{+}+\mathbf{R}^{\cap} \mathbf{P}^{-}\right] \vec{\Gamma}_{j}$. The secondary sources improve the illumination of the subsurface, particularly in areas where primary source wavefields are very weak.

In practice, the blended double-illuminating wavefield is known at the positions where the primary sources are present (given by the source elements in $\mathbf{S}^{+} \vec{\Gamma}_{j}$ ) and for the positions where the secondary sources are measured (i.e., the detector locations given by the elements in $\left.\mathbf{R}^{\cap} \mathbf{P}^{-} \vec{\Gamma}_{j}\right)$. Therefore, the benefits to be obtained from the secondary illumination depend on the detector distribution of the acquisition geometry.

In high-reflectivity areas, the secondary sources $\left(\mathbf{R}^{\cap} \vec{P}_{j}^{-}\right)$will give an important contribution to the stabilization of shot-record migration algorithms. This is particularly true if - for economic or 
practical reasons - significant gaps in the offset or azimuth distribution occur.

\section{APPENDIX A}

\section{MATRIX REPRESENTATION OF WAVE THEORY}

Throughout this paper, the matrix notation is used as introduced by Berkhout (1982). In this appendix, the matrices, vectors (matrix rows and columns), and matrix elements that appear in the paper are explained in more detail. We start with the data matrix $\mathbf{P}\left(z_{d}, z_{s}\right)$. Every column of $\mathbf{P}\left(z_{d}, z_{s}\right)$ represents a common source gather (shot record), every row represents a common detector gather, and spatial acquisition parameters $z_{d}$ and $z_{s}$ refer to the detector and source depth levels, respectively. Every element of $\mathbf{P}\left(z_{d}, z_{s}\right)$ corresponds to a single trace of a seismic data volume. It represents either one time series or one frequency component. In the latter case the matrix elements are complex-valued scalars. In this Appendix, we consider the matrix notation in the frequency domain and discuss the matrices for one frequency (one frequency slice of the data volume). Note that frequency slices are orthogonal and therefore may be treated independently. Note also that processing of frequency slices allows a multiscale approach.

In the case that the seismic experiment is carried out at (or close to) surface $z_{0}$, i.e., $z_{s}=z_{d}=z_{0}$, the data matrix becomes $\mathbf{P}\left(z_{0}, z_{0}\right)$. For notational simplicity the depths of the sources and detectors are sometimes omitted, such that $\mathbf{P}\left(z_{0}, z_{0}\right)$ or $\mathbf{P}\left(z_{d}, z_{s}\right)$ is referred to as $\mathbf{P}$. Note that data matrix $\mathbf{P}$ always has two dimensions, rows and columns, corresponding to the $\left(x_{s}, y_{s}\right)$ source positions (columns) and the $\left(x_{d}, y_{d}\right)$ detector positions (rows), respectively, irrespective of the dimensionality of the seismic survey which may be 2D or 3D.

Column $j$ of matrix $\mathbf{P}$ is indicated by vector $\vec{P}_{j}$. Column vector $\vec{P}_{j}$ corresponds to the $j$ th shot record. Row $i$ of $\mathbf{P}$, which corresponds to the $i$ th common detector gather, is indicated by $\vec{P}_{i}^{\dagger}$. In our notation, the dagger symbol $\dagger$ is used to denote a row vector. Element $i, j$ of matrix $\mathbf{P}$ is denoted as $P_{i j}$; it corresponds to one frequency component of the seismic trace generated by source $j$ and recorded by detector $i$. Note that source and detector may also be interpreted as source array (think of vibrator group or airgun array) and detector array (think of geophone pattern or hydrophone group), respectively.

If it is desired to make a distinction between the propagation direction of the wavefield, we denote downward traveling wavefields by $\mathbf{P}^{+}$and upward traveling wavefields by $\mathbf{P}^{-}$.

Apart from matrix $\mathbf{P}$, we also clarify the following matrices (equation 1): $\mathbf{S}^{+}, \mathbf{R}^{\bigcap}$, and $\mathbf{X}_{0}$. Matrix $\mathbf{S}^{+}$represents the source matrix. The superscript in $\mathbf{S}^{+}$refers to a downgoing wavefield. One source (array) $j$ is described by column $j$ of $\mathbf{S}^{+}$, which is denoted as $\vec{S}_{j}^{+}$. Column vector $\vec{S}_{j}^{+}\left(z_{s}\right)$ describes the source wavefield at source level $z_{s}$. The locations of its elements $S_{i j}^{+}\left(z_{s}\right)$ in the column correspond to the lateral locations of the source array. In the case of a single dipole source, one element of $\vec{S}_{j}^{+}\left(z_{s}\right)$ has a nonzero value, its location in the column corresponding to the dipole location. In the case of a source array, several elements of $\vec{S}_{j}^{+}\left(z_{s}\right)$ may have a nonzero value, their locations in the column corresponding to the lateral locations of the sources forming the array. For a traditional source array, the individual sources within such an array are located closely together and they are activated (almost) simultaneously. This, in contrast with the sources of a blended source array, which may be far apart and activated with considerable temporal intervals between them.

Matrix $\mathbf{X}_{0}\left(z_{d}, z_{s}\right)$ is the earth's transfer function without surfacerelated multiples. Every column contains an impulse response of the earth: from a single source location at level $z_{s}$, via downward propagation, reflection, and upward propagation to detector locations at level $z_{d}$. Note that the model (equation 1) is very general: It doesn't show the detail of such an impulse response. It states that for a model that is linear in the wavefields, the earth changes the amplitude and phase of a frequency component of the source wavefield while it is traveling from the source location to the detector locations (bear in mind that it does not generate other frequency components). In practice, the elements of $\mathbf{X}_{0}$ may be computed using ray-tracing, finite-difference modeling, finite-element modeling, or any integral modeling method (Berkhout, 2010).

Matrix $\mathbf{R}^{\cap}$ represents the surface reflectivity. It turns an upward traveling wavefield into a downward traveling wavefield, indicated by the superscript $\cap$. Every column contains the angle-dependent reflection information of one surface location. In the case of angle-independent reflectivity, $\mathbf{R}^{\cap}$ is a diagonal matrix, meaning that every column has one nonzero element which equals the reflection coefficient of the corresponding surface location. Note that for seismic frequencies the water surface can be described by a diagonal matrix $\mathbf{R}^{\cap}$ which elements equal -1 , i.e., $\mathbf{R}^{\cap}=-\mathbf{I}$. This means that the reflection is total, angle-independent, and the polarity is changed (phase rotation of $180^{\circ}$ ). In the case of an angle-dependent surface reflectivity, $\mathbf{R}^{\cap}$ becomes a band matrix.

In equation 6 we make use of column vector $\vec{\Gamma}_{j}\left(z_{0}\right)$, row vector $\vec{W}_{k}^{\dagger}\left(z_{m}, z_{0}\right)$, and matrix element $P_{k j}^{+}\left(z_{m}, z_{0}\right)$. Column vector $\vec{\Gamma}_{j}$ is a blending vector. It provides the blending information of blended source array $j . \vec{\Gamma}_{j}$ is the $j$ th column of blending matrix $\boldsymbol{\Gamma}$ which contains the blending information of all blended source arrays in a seismic survey. Each element $\Gamma_{i j}$ contains the blending code to be applied to the corresponding source column $\vec{S}_{i}^{+}$, after which the coded sources are summed (matrix-vector multiplication $\mathbf{S}^{+} \vec{\Gamma}_{j}=\sum_{i} \vec{S}_{i}^{+} \Gamma_{i j}$ ). Note that the code may be a simple time shift. In that case, element $\Gamma_{i j}$ equals $\exp \left(-j \omega \tau_{i j}\right)$, where $\tau_{i j}$ is the corresponding time shift.

The downward propagation of the total illuminating wavefield from all surface locations to all gridpoins at depth level $z_{m}$ is given by the propagation matrix $\mathbf{W}\left(z_{m}, z_{0}\right)$. Propagation to gridpoint $k$ at depth level $z_{m}$, see equation 6 , is carried out via multiplication with row vector $\vec{W}_{k}^{\dagger}\left(z_{m}, z_{0}\right)$. Its elements $W_{k j}\left(z_{m}, z_{0}\right)$ contain the phase and amplitude information related to the downward propagation from all surface locations $\left(x_{j}, y_{j}, z_{0}\right)$ to single subsurface location $\left(x_{k}, y_{k}, z_{m}\right)$. Again, the formulation is general: It doesn't show the details of $\vec{W}_{k}^{\dagger}$, nor does it state how $\vec{W}_{k}^{\dagger}$ should be computed - by ray tracing, finite-difference modeling, etc. — but it makes clear that subsurface gridpoint $k$ receives the sum of contributions of the sources at the surface.

The incident wavefield in a subsurface gridpoint is a virtual seismic trace (as if a detector were located at that gridpoint), see equation 6 . Therefore, in the frequency domain, it is denoted by a complex-valued scalar $P_{k j}^{+}\left(z_{m}, z_{0}\right)$. As mentioned, the superscript refers to a downgoing wavefield. The wavefield is a doubleilluminating wavefield, received at subsurface gridpoint $k$ of depth level $z_{m}$ and generated by blended primary source array $\mathbf{S}^{+} \vec{\Gamma}_{j}^{+}$and 
blended-secondary source array $\mathbf{R}^{\cap} \mathbf{P}^{-} \vec{\Gamma}_{j}^{+}$, both being located at surface $z_{0}$.

\section{REFERENCES}

Akerberg, P., G. Hampson, J. Rickett, H. Martin, and J. Cole, 2008, Simultaneous source separation by sparse radon transform: 78th Annual International Meeting, SEG, Expanded Abstracts, 27, 2801-2805.

Bagaini, C., 2006, Overview of simultaneous vibroseis acquisition methods: 78th Annual International Meeting, SEG, Expanded Abstracts, 25, 70-74.

Beasley, C. J., 2008, A new look at marine simultaneous sources: The Leading Edge, 27, 914-917, doi: 10.1190/1.2954033.

Beasley, C. J., R. E. Chambers, and Z. Jiang, 1998, A new look at simultaneous sources: 68th Annual International Meeting, SEG, Expanded Abstracts, 17, 133-135.

Beasley, C. J., E. Saragoussi, S. Whidden, and F. L. Diagon, 2010, Faster, cheaper, better seismic acquisition: Now you can have all three: 80th Annual International Meeting, SEG, Expanded Abstracts, 29, 3807-3810.

Berkhout, A. J., 1982, Seismic migration, imaging of acoustic energy by wave field extrapolation, A. theoretical aspects: Elsevier.

Berkhout, A. J., 1997, Pushing the limits of seismic imaging, part II: integration of prestack migration, velocity estimation and AVO analysis: Geophysics, 62, 954-969, doi: 10.1190/1.1444202.

Berkhout, A. J., 2008, Changing the mindset in seismic data acquisition: The Leading Edge, 27, 924-938, doi: 10.1190/1.2954035.

Berkhout, A. J., 2010, Parameterization of seismic data using gridpoint responses: 80th Annual International Meeting, SEG, Expanded Abstracts, 29, 3344-3348.

Berkhout, A. J., and D. J. Verschuur, 1994, Multiple technology, part 2: Migration of multiple reflections: 64th International Meeting, SEG, Expanded Abstracts, 13, 1497-1500.

Bostock, M., S. Rondenay, and J. Shragge, 2001, Multiparameter twodimensional inversion of scattered teleseismic body waves 1 . Theory for oblique incidence: Journal of Geophysical Research, 106, 3077130782, doi: 10.1029/2001JB000330.

Hampson, G., J. Stefani, and F. Herkenhoff, 2008, Acquisition using simultaneous sources: The Leading Edge, 27, 918-923, doi: 10.1190/ 1.2954034

Howe, D., M. Foster, T. Allen, B. Taylor, and I. Jack, 2008, Independent simultaneous sweeping - A method to increase the productivity of land seismic crews: 78th Annual International Meeting, SEG, Expanded Abstracts, 27, 2826-2830.
Huo, S., Y. Luo, and P. Kelamis, 2009, Simultaneous sources separation via multi-directional vector-median filter: 79th Annual International Meeting, SEG, Expanded Abstracts, 28, 31-35.

Ikelle, L., 2007, Coding and decoding: Seismic data modeling, acquisition and processing: 77th Annual International Meeting, SEG, Expanded Abstracts, 26, 66-70.

Jiang, Z., J. Yu, G. T. Schuster, and B. E. Hornby, 2005, Migration of multiples: The Leading Edge, 24, 315-318, doi: 10.1190/1.1895318.

Kim, Y., I. Gruzinov, M. Guo, and S. Sen, 2009, Source separation of simultaneous source OBC data: 79th Annual International Meeting, SEG, Expanded Abstracts, 28, 51-55.

Lin, T. T. Y., and F. J. Herrmann, 2009, Designing simultaneous acquisitions with compressive sensing: Presented at the 71st Annual International Conference and Exhibition, EAGE

Moore, I., B. Dragoset, T. Ommundsen, D. Wilson, C. Ward, and D. Eke, 2008, Simultaneous source separation using dithered sources: 78th Annual International Meeting, SEG, Expanded Abstracts, 27, 2806-2810.

Pecholcs, P. I., S. K. Lafon, T. Al-Ghamdi, H. Al-Shammery, P. G. Kelamis, S. X. Huo, O. Winter, J.-B. Kerboul, and T. Klein, 2010, Over 40,000 vibrator points per day with realtime quality control: Opportunities and challenges: 80th Annual International Meeting, SEG, Expanded Abstracts, 29, 111-115.

Rondenay, S., M. Bostock, and K. Fisher, 2005, Multichannel inversion of scattered teleseismic body waves: Practical considerations and applicability in Seismic Earth: Array analysis of broadband seismograms: A. Levander, and G. Nolet eds.,AGU, 157, 187-203.

Schuster, G., 2005, Fermat's interferometric principle for multiple reflection tomography: Geophysical Research Letters, 32, no. 12, L12303, doi 10.1029/2005GL022351.

Spitz, S., G. Hampson, and A. Pica, 2008, Simultaneous source separation: A prediction subtraction approach: 78th Annual International Meeting, SEG, Expanded Abstracts, 2811-2814.

Tabti, H., A. Day, T. Schade, M. Lesnes, and T. Hy, 2009, Conventional versus dual-sensor streamer data de-ghosting — Acase study from the Haltenbanken dual-streamer acquisition: 79th Annual International Meeting, SEG, Expanded Abstracts, 28, 137-141, doi: 10.1190/1.3255106.

Taner, M., 1976, Simplan: Simulated plane wave exploration: 46th Annual International Meeting, SEG, Expanded Abstracts, 186-187.

Vaage, S. T., 2002, Method and system for acquiring marine seismic data using multiple seismic sources: U.S. Patent 6906981 .

Verschuur, D. J., and A. J. Berkhout, 2011, Seismic migration of blended shot records with surface-related multiple scattering: Geophysics, 76, no. 1, A7-13.

Whitmore, N. D., A. A. Valenciano, and W. Sollner, 2010, Imaging of primaries and multiples using a dual-sensor towed streamer: 80th Annual international Meeting, SEG, Expanded Abstracts, 29, 3187-3192. 